\title{
Convergence and Performance Issues for Autocorrelation Based Adaptive Channel Shortening
}

\author{
(Invited Paper)
}

\author{
John MacLaren Walsh \\ Dept. of Elec. and Comp. Eng. \\ Drexel University \\ Philadelphia, PA 19104 \\ Email: jwalsh@coe.drexel.edu
}

\author{
Richard K. Martin \\ Dept. of Elec. and Comp. Eng. \\ Air Force Institute of Technology \\ WPAFB, OH 45433 \\ Email: Richard.Martin@afit.edu
}

\author{
C. Richard Johnson, Jr. \\ School of Elec. and Comp. Eng. \\ Cornell University \\ Ithaca, NY 14853 \\ Email: johnson@ece.cornell.edu
}

\begin{abstract}
We derive bounds on the signal to interference ratio performance of several blind channel shortening designs based on autocorrelation minimization. We focus in particular on the performance of these designs in oversampled communications systems with multiple receive antennas. The utility of the bounds is demonstrated by proving inherent performance problems with certain classes of designs that previous low dimensional plots of the cost functions were not able to predict. We conclude the paper by providing a convergence result for adaptive versions of these blind designs which can be coupled with the performance results to determine performance of the adaptive designs.
\end{abstract}

\section{INTRODUCTION}

Channel shortening has been widely discussed as a method to limit the computational complexity of the use of Viterbi maximum likelihood sequence detection in single carrier communication systems [1] as well as a method to avoid cyclic prefix length violations in multi-carrier communications systems [2]. Blind channel shortening refers to situations in which the channel shortener must be designed without knowledge of the transmission of a pre-arranged "training" sequence . Blind designs are relevant when no such training signal is sent in order to increase transmission capacity or, alternatively, when a training signal is sent, but the recipient is unaware of of it, as in signal intelligence applications. In this paper, we quantify the signal to interference ratio performance of several blind channel shortening designs which derive error signals using the auto-correlation of the received signal. In particular, we focus on the sum squared auto-correlation minimization (SSAM) [4], sum absolute auto-correlation minimization (SAAM) [5], and single lag auto-correlation minimization (SLAM) [6] designs. We explain the importance of the use of a matched filter in systems employing such designs, although it remains not entirely clear how such a matched filter is to be implemented in practice given the blind constraint. The bounds show that the SLAM designs can suffer from performance problems which

R. K. Martin was funded in part by the Air Force Office of Scientific Research. The views expressed in this paper are those of the authors, and do not reflect the official policy or position of the United States Air Force, Department of Defense, or the U.S. Government. This document has been approved for public release; distribution unlimited.

C. R. Johnson, Jr. was supported in part by Applied Signal Technology and NSF grant 0310023. previous low dimensional plots of the cost functions were not able to predict, while SSAM and SAAM designs can be expected to perform comparably to trained designs provided a matched filter or a minimum phase constraint is possible.

\section{Notation And System Model}

In this paper, we consider the use of a channel shortening filter at the output of a channel, as shown in Figure 1. The case in Figure 1 where $\mathrm{L}>1$ is referred to as a single input multiple output channel. This SIMO channel can arise from either from over-sampling at the receiver or by the use of multiple receive antennas. Either way, the received signal at the input to the receiver is a vector valued signal $r_{\mathrm{n}}:=\left[r_{\mathrm{n}}^{(1)}, r_{\mathrm{n}}^{(2)}, \ldots, r_{\mathrm{n}}^{(\mathrm{L})}\right]$, which is the sum of some additive noise $\sigma_{\mathrm{n}}:=\left[\sigma_{\mathrm{n}}^{(1)}, \sigma_{\mathrm{n}}^{(2)}, \ldots, \sigma_{\mathrm{n}}^{(\mathrm{L})}\right]$ and the output signal from the transmitter $s_{\mathrm{n}}$ filtered by a channel filter $\mathrm{h}(z):=$ $\left[\mathrm{h}^{(1)}(z), \mathrm{h}^{(2)}(z), \ldots, \mathrm{h}^{(\mathrm{L})}(z)\right]^{\dagger}$ (where $\dagger$ denotes the transpose operation). Here, each sub-channel $\mathrm{h}^{(\mathrm{i})}(z)$ is modeled as a finite impulse response filter of order $\mathrm{M}$

$$
\mathrm{h}^{(\mathrm{i})}(z):=\sum_{\mathrm{n}=0}^{\mathrm{M}} h_{\mathrm{n}}^{(\mathrm{i})} z^{-\mathrm{n}}
$$

At the receiver, the vector valued input $r_{\mathrm{n}}$ is processed by a channel shortening filter $\mathrm{w}(z):=$ $\left[\mathrm{w}^{(1)}(z), \mathrm{w}^{(2)}(z), \ldots, \mathrm{w}^{(\mathrm{L})}(z)\right]$ which sums the output of channel shortening filters $\mathrm{w}^{(\mathrm{i})}(z)$ operating on each of the sub-channel outputs $r_{\mathrm{n}}^{(\mathrm{i})}$. We shall consider channel shortening filters with impulse responses of order K, so that

$$
\mathrm{w}^{(\mathrm{i})}(z):=\sum_{\mathrm{n}=0}^{\mathrm{K}} w_{\mathrm{n}}^{(\mathrm{i})} z^{-\mathrm{n}}
$$

The overall effective filter between the transmitted symbols $s_{\mathrm{n}}$ and the output of the channel shortener $y_{\mathrm{n}}$ may then be written as

$$
\mathrm{c}(z):=\mathrm{w}^{\dagger}(z) \mathrm{h}(z):=\sum_{\mathrm{i}=1}^{\mathrm{L}} \mathrm{w}^{(\mathrm{i})}(z) \mathrm{h}^{(\mathrm{i})}(z)
$$




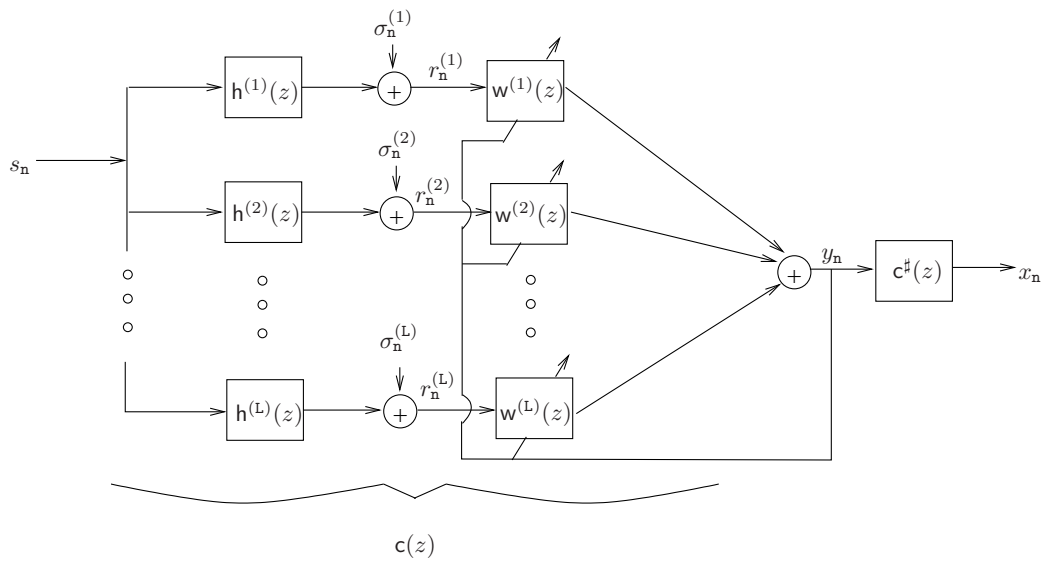

Fig. 1. System Model.

The receiver then processes this output $y_{\mathrm{n}}$ with a matched filter

$$
c^{\sharp}(z):=\sum_{\mathrm{n}=0}^{\mathrm{N}} c^{*} z^{\mathrm{n}}=\mathrm{c}^{*}\left(\left(z^{-1}\right)^{*}\right)
$$

where $*$ denote complex conjugation, to create the final output $x_{\mathrm{n}}$ which is passed on to the remainder of the receiver.

The goal of the channel shortening filter is the ensure that final transfer function $c^{\sharp}(z) c(z)$ between the output of the transmitter and the output of the matched filter has an impulse response that is zero outside of a window of length $2 \nu+1$. In single carrier systems this can be due the use of a optimal Viterbi or forward-backward sequence detector at the output of the matched filter. Since the complexity of these optimal sequence detection algorithms scales exponentially in the effective impulse response length, it is often computationally infeasible to use them for long effective impulse responses. In these instances, the channel shortener allows one to reap the performance benefits of the Viterbi or Baum-Welch optimal sequence detection algorithms at a reasonable computational complexity by shortening the length of the effective channel. In multicarrier systems such as DMT or C-OFDM, on the other hand, one employs the channel shortener to ensure that only simple scalar equalization is required one each bin the output of the FFT. We will not assume either of these instances in particular. Instead, we shall only assume that the signal $s_{\mathrm{n}}$ has zero mean, unit-variance, and is uncorrelated, so that $\mathbb{E}\left[s_{\mathrm{n}} s_{\mathrm{n}^{\prime}}\right]=0$ for $\mathrm{n} \neq \mathrm{n}^{\prime}$. We shall further assume that the sub-channels $\mathrm{h}^{(\mathrm{i})}(z)$ share have no common zeros (i.e. are co-prime), and the length of the shortening filters has been selected in a manner so that any effective transfer function $\mathrm{c}(z)$ can be created by choosing an appropriate channel shortener. Length conditions for this to hold, as well as relaxation of the co-prime requirement, were discussed in [7], [8]. Finally, we will focus on the interference dominated regime, so that the noise process $\sigma_{\mathrm{n}}$, may be neglected.

\section{Blind Adaptive Channel Shortening Metrics}

In this section, we review the blind channel shortening metrics of interest. By metric, we mean a function which assigns to every combined response $c(z)$ a cost. A channel shortening design according to a particular metric corresponds to the combined response $\mathrm{c}(z)$ with the minimum metric that is achievable for some shortener $\mathrm{w}(z)$. Our study will be focussed on channel shortening designs which operate using the auto-correlation of $y_{\mathrm{n}}$, and thus on the autocorrelation of the combined response $\mathrm{c}(z)$, whose transform may be written as

$$
\mathrm{q}(z):=\mathrm{c}(z) \mathrm{c}^{\sharp}(z)=\sum_{\mathrm{n}=-\mathrm{N}}^{\mathrm{N}} q_{\mathrm{n}} z^{-\mathrm{n}}
$$

The metrics we consider all require an extra constraint, which we choose to be a unit energy constraint on $\mathrm{c}(z)$, so that

$$
\sum_{\mathrm{n}=0}^{\mathrm{N}}\left|c_{\mathrm{n}}\right|^{2}=q_{0}=1
$$

The sum squared autocorrelation metric (SSAM) [4] is simply the sum of the autocorrelation squared outside of the window of length $2 \nu+1$.

$$
\mathrm{J}_{S}:=\sum_{|\mathrm{k}| \geq \nu+1}\left|q_{\mathrm{m}}\right|^{2}=2 \sum_{\mathrm{k}=\nu+1}^{\mathrm{N}}\left|q_{\mathrm{m}}\right|^{2}
$$

Similarly, the sum absolute autocorrelation metric (SAAM) [5] is simply the sum of the absolute autocorrelation values outside of a window of length $2 \nu+1$

$$
\mathrm{J}_{A}:=\sum_{|\mathrm{k}| \geq \nu+1}\left|q_{\mathrm{m}}\right|
$$

Finally, the single lag autocorrelation metric (SLAM) [6] attempts to reduce the complexity of SAM designs by minimizing the absolute value of only the correlation at the $\nu+1$ th delay.

$$
\mathrm{J}_{L}:=\left|q_{\nu+1}\right|^{2}
$$


It is not to difficult to prove that $\mathrm{J}_{S}, \mathrm{~J}_{A}$, and $\mathrm{J}_{L}$ are all zero if the combined response $c(z)$ has taps which are all zero except for possibly some within a window of length $\nu$ [4][5][6]. The non-negative definiteness of these metrics then shows that they all have global minima for shortened $\mathrm{c}(z)$. Furthermore, $\mathrm{J}_{S}$ and $\mathrm{J}_{A}$ are equal to zero only if $\mathrm{c}(z)$ has taps which are all zero except possibly within a window of size $\nu+1$. Thus, for SSAM and SAAM the global minima are all at perfectly shortened channels. As we shall see later, however, the same is not true of the SLAM cost. These global minima (partially) establish the utility of the SSAM, SAAM, and SLAM costs. Before we move further, it is important to note that these designs suffer from inherent ambiguities in terms of the combined response $\mathrm{c}(z)$ simply because they depend on $\mathrm{c}(z)$ only through the auto-correlation $\mathrm{q}(z)$. In particular the auto-correlation of a combined response $\mathrm{c}(z)$ remains unchanged if one replaces a zero by its conjugate inverse and re-normalizes to enforce the unit energy constraint. To see why this is the case, let the zeros of $c(z)$ be $\left\{d_{\mathrm{n}}\right\}$, so that

$$
\mathrm{c}(z):=a_{0} \prod_{\mathrm{n}=1}^{\mathrm{N}}\left(1-d_{\mathrm{n}} z^{-1}\right)
$$

This gives an autocorrelation with transform

$$
\mathrm{c}(z) \mathrm{c}^{\sharp}(z)=\left|a_{0}\right|^{2} \prod_{\mathrm{n}=1}^{\mathrm{N}}\left(1-d_{\mathrm{n}} z^{-1}\right)\left(1-d_{\mathrm{n}}^{*} z\right)
$$

Now consider $c_{2}(z)$, which is created by flipping one of the zeros over the unit circle and conjugating it, i.e. by replacing $d_{1}$ by $\frac{1}{d_{1}^{*}}$, and then normalizing the taps so that they are unit norm, so that

$$
\mathrm{c}_{2}(z)=b_{0}\left(1-\frac{z^{-1}}{d_{1}^{*}}\right) \prod_{\mathrm{n}=2}^{\mathrm{N}}\left(1-d_{\mathrm{n}} z^{-1}\right)
$$

Then $c_{2}(z)$ has an autocorrelation with transform

$$
\begin{aligned}
\mathrm{c}_{2}(z) \mathrm{c}_{2}^{\sharp}(z)= & \left|b_{0}\right|^{2}\left(1-\frac{z^{-1}}{d_{1}^{*}}\right)\left(1-d_{1}^{-1} z\right) \\
& \prod_{\mathrm{n}=2}^{\mathrm{N}}\left(1-d_{\mathrm{n}} z^{-1}\right)\left(1-d_{\mathrm{n}}^{*} z\right) \\
= & \frac{\left|b_{0}\right|^{2}}{\left|d_{1}\right|^{2}} \prod_{\mathrm{n}=1}^{\mathrm{N}}\left(1-d_{\mathrm{n}} z^{-1}\right)\left(1-d_{\mathrm{n}}^{*} z\right) \\
= & \mathrm{c}(z) \mathrm{c}^{\sharp}(z)
\end{aligned}
$$

where the last equality followed from $\frac{\left|b_{0}\right|^{2}}{\left|d_{1}\right|^{2}}=\left|a_{0}\right|^{2}$ due to the unit energy constraint. This brings us to our next point, which concerns the importance of the inclusion of the matched filter $c^{\sharp}(z)$ in the system, as in Figure 1 .

\section{A. Importance of the Matched Filter}

Let us assume for the moment that the matched filter $c^{\sharp}(z)$ was not included in Figure 1, so that the signal output to the rest of the (not pictured) receiver chain was $y_{n}$. Without the matched filter, the goal becomes to shorten the channel to $\nu$ non-zero taps. Let us quantify the performance of this system with the matched filter removed by the best delay signal to inference ratio

$$
\operatorname{SIR}\left(\left\{y_{\mathrm{n}}\right\}\right):=\max _{\Delta} \frac{\sum_{\mathrm{n}=\Delta}^{\Delta+\nu}\left|\mathbf{c}_{\mathrm{n}}\right|^{2}}{\sum_{\mathrm{n}=0}^{\Delta-1}\left|\mathbf{c}_{\mathrm{n}}\right|^{2}+\sum_{\mathrm{n}=\Delta+\nu+1}^{\mathrm{N}}\left|\mathbf{c}_{\mathrm{n}}\right|^{2}}
$$

It turns out that the SSAM, SAAM, and SLAM costs are unsuited for this system, because there are combined responses $c(z)$ with costs very near to the global optimal value (0) of these costs with terrible SIR. This is all due to the autocorrelation based nature of the SAAM, SSAM, and SLAM designs. In particular, unlike the auto-correlation, the quality of a combined response as a channel shortener (e.g. its SIR) changes incredibly when you flip one of its zeros over the unit circle and conjugate it.

This point is perhaps best illustrated with an example. Consider a combined response $\mathrm{c}(z)$ whose zeros $d_{\mathrm{n}}, \mathrm{n} \in$ $\{1, \ldots, N\}$ are $(\jmath:=\sqrt{-1})$

$$
d_{\mathrm{n}}=\alpha \exp \left(\jmath \frac{2 \pi \mathrm{n}}{\mathrm{N}}\right), \mathrm{n} \in\{1, \ldots, \mathrm{N}\}
$$

This gives a combined response, after unit energy normalization, of

$$
c(z)=\frac{1}{\sqrt{1+\alpha^{2 \mathrm{~N}}}}-\frac{\alpha^{\mathrm{N}}}{\sqrt{1+\alpha^{2 \mathrm{~N}}}} z^{-\mathrm{N}}
$$

which, for $\alpha<1$ will have a best-delay SIR of $-20 \mathrm{~N} \log _{10}(\alpha)$ $\mathrm{dB}$, which can be made arbitrarily large via choice of $\alpha$. As one would expect, the SSAM, SAAM, and SLAM costs for this response are very low as well. In particular, the SSAM cost is $10 \log _{10}\left(\frac{\alpha^{2 \mathrm{~N}}}{\left(1+\alpha^{2 \mathrm{~N}}\right)^{2}}\right) \mathrm{dB}$ and the SAAM cost is $10 \log _{10}\left(\frac{\alpha^{\mathrm{N}}}{\left(1+\alpha^{2 \mathrm{~N}}\right)}\right)$ for any $\nu$. The SLAM cost is $-\infty \mathrm{dB}$ for any $\nu<\mathrm{N}$, and is $10 \log _{10}\left(\frac{\alpha^{\mathrm{N}}}{\left(1+\alpha^{2 \mathrm{~N}}\right)}\right)$ for $\nu=\mathrm{N}$. Because they depend only on the autocorrelation, the SSAM, SAAM, and SLAM costs do not change if we make the changes

$$
d_{1} \mapsto \frac{1}{d_{1}}, d_{\mathrm{N}-1} \mapsto \frac{1}{d_{\mathrm{N}-1}}, d_{\mathrm{N}} \mapsto \frac{1}{d_{\mathrm{N}}}
$$

The best delay SIR, however, changes drastically under this transformation. The particular instance when $\alpha=\frac{1}{2}, \nu=1$, and $\mathrm{N}=9$ is shown in Figure 2. Here the best delay SIR was $54 \mathrm{~dB}$ before the translation (1), but after the translation 1 the best delay SIR becomes $1 \mathrm{~dB}$. The SSAM and SAAM costs remain at $-54 \mathrm{~dB}$ and $-27 \mathrm{~dB}$ respectively during this translation. The SLAM cost remains at $-\infty \mathrm{dB}$.

If, however, a matched filter is added to the receiver, then the effective impulse response between $s_{\mathrm{n}}$ and $x_{\mathrm{n}}$ is the autocorrelation of $\mathrm{c}(z)$, i.e. $\mathrm{c}(z) \mathrm{c}^{\sharp}(z)$. This means that the SSAM and SAAM costs are minimizing the sum squared magnitude and the sum magnitude, of the taps outside of the window centered at zero (now of length $2 \nu+1$ ) in the effective response $\mathrm{q}(z)$ between $s_{\mathrm{n}}$ and $x_{\mathrm{n}}$, which is directly related to the signal to interference ratio of $x_{\mathrm{n}}$, as we shall show via a bounding argument in the next section. 

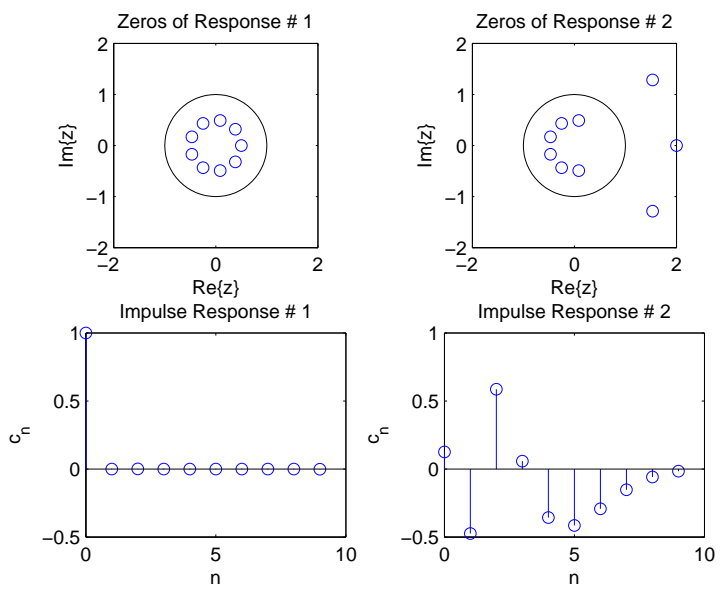

Fig. 2. Two combined responses $c(z)$ with the same autocorrelation, and thus the same SSAM, SAAM, and SLAM costs $(-54 \mathrm{~dB},-27 \mathrm{~dB}$, and $-\infty$ $\mathrm{dB}$, respectively), but with very different best delay SIRs when no matched filter is used $(54 \mathrm{~dB}$ versus $1 \mathrm{~dB})$.

At this point it should be noted that, although it is clear from the preceding argument and example that a matched filter is necessary for blind designs based on the autocorrelation of the combined response, it is not entirely clear how one may go about determining the appropriate matched filter at the receiver. In particular, as pointed out in [3], [4], [5], [6], one can make adaptive channel shortening filters which adaptively minimize these costs directly via choice of the channel shortener $w(z)$. Because these algorithms operate directly on the received data without ever estimating the channel $\mathrm{h}(z)$ or the combined response $\mathrm{c}(z)$, after they have converged, although the channel may be shortened, the combined impulse response remains unknown. Thus, the requirement of a matched filter also implicitly includes the requirement that the combined impulse response $\mathrm{c}(z)$ be estimated. Alternatively, the example provided above seems to suggest that a minimum phase requirement on $\mathrm{c}(z)$ may be sufficient, although this again may require estimation of $\mathrm{c}(z)$ in order to determine if it is minimum phase. Either way, it seems likely that $\mathrm{c}(z)$ will have to be estimated, or auto-correlation based designs will suffer from the ambiguities indicated above.

\section{BOUNDS ON THE SIR PERFORMANCE OF Autocorrelation Based Designs}

We now return to the system depicted in Figure 1 with the matched filter present, and we wish to provide a relation between the blind channel shortening metrics SSAM, SAAM, and SLAM and the signal to inference power ratio in $x_{\mathrm{n}}$, which we define to be

$$
\mathrm{SIR}:=\frac{\sum_{\mathrm{m}=-\nu}^{\nu}\left|q_{\mathrm{m}}\right|^{2}}{\sum_{\mathrm{m}=-\mathrm{N}}^{-\nu+1}\left|q_{\mathrm{m}}\right|^{2}+\sum_{\mathrm{m}=\nu+1}^{\mathrm{N}}\left|q_{\mathrm{m}}\right|^{2}}
$$

Recognizing the denominator in this expression as the SSAM cost, and considering only those $\mathrm{c}(z)$ which satisfy the unit energy constraint, we may immediately obtain the relation

$$
\begin{aligned}
\operatorname{SIR}(\mathrm{dB}) & =10 \log _{10}\left(\sum_{\mathrm{m}=-\nu}^{\nu}\left|q_{\mathrm{m}}\right|^{2}\right)-10 \log _{10}\left(\mathrm{~J}_{S}\right) \\
& =10 \log _{10}\left(1+2 \sum_{\mathrm{m}=1}^{\nu}\left|q_{\mathrm{m}}\right|^{2}\right)-10 \log _{10}\left(\mathrm{~J}_{S}\right) \\
& \geq-\mathrm{J}_{S}(\mathrm{~dB})
\end{aligned}
$$

So that a low SSAM cost can be guaranteed to give a high SIR at the output of the matched filter. Furthermore, since

$$
\begin{aligned}
\mathrm{J}_{A}^{2} & =\sum_{|\mathrm{m}| \geq \nu}\left|q_{\mathrm{m}}\right|^{2}+\sum_{|\mathrm{i}| \geq \nu} \sum_{|\mathrm{n}| \geq \nu, \mathrm{n} \neq \mathrm{i}}\left|q_{\mathrm{m}}\right|\left|q_{\mathrm{i}}\right| \\
& =J_{S}+\sum_{|\mathrm{i}| \geq \nu} \sum_{|\mathrm{n}| \geq \nu, \mathrm{n} \neq \mathrm{i}}\left|q_{\mathrm{m}}\right|\left|q_{\mathrm{i}}\right| \\
& \geq J_{S}
\end{aligned}
$$

we may also underbound the performance with the SAAM cost

$$
\operatorname{SIR}(\mathrm{dB}) \geq-2 \mathrm{~J}_{A}(\mathrm{~dB})
$$

So that a low SAAM cost also guarantees a high SIR at the output of the matched filter. Unfortunately, for a reason we shall discuss in the next section, the SLAM design affords no such underbound on the performance SIR.

Moving now to upper bounds, one may note that for $\mathrm{c}(z)$ satisfying the unit energy constraint, $\mathrm{m}>0$,

$$
\begin{aligned}
& \left|q_{\mathrm{m}}\right| \leq \quad \underset{\mid \text { eigenvalue }}{\text { maximum }} \quad \frac{1}{2}\left[\begin{array}{cc}
\mathbf{0}_{(\mathrm{N}+1-\mathrm{m}) \times \mathrm{m}} & \mathbf{I}_{\mathrm{N}+1-\mathrm{m}} \\
\mathbf{0}_{\mathrm{m} \times \mathrm{m}} & \mathbf{0}_{\mathrm{m} \times(\mathrm{N}+1-\mathrm{m})}
\end{array}\right] \\
& +\frac{1}{2}\left[\begin{array}{cc}
\mathbf{0}_{\mathrm{m} \times(\mathrm{N}+1-\mathrm{m})} & \mathbf{0}_{\mathrm{m} \times \mathrm{m}} \\
\mathbf{I}_{\mathrm{N}+1-\mathrm{m}} & \mathbf{0}_{(\mathrm{N}+1-\mathrm{m}) \times \mathrm{m}}
\end{array}\right]
\end{aligned}
$$

Denoting this maximum eigenvalue magnitude by $\left|\lambda_{\mathrm{MAX}, \mathrm{m}, \mathrm{N}}\right|$, we may upper bound the SIR at the matched filter output among those $\mathrm{c}(z) \mathrm{s}$ obeying the unit energy constraint by

$$
\operatorname{SIR}(\mathrm{dB}) \leq 10 \log _{10}\left(1+2 \sum_{0<\mathrm{m} \leq \nu}\left|\lambda_{\mathrm{MAX}, \mathrm{m}, \mathrm{N}}\right|^{2}\right)-\mathrm{J}_{S}(\mathrm{~dB})
$$

Furthermore, since via the relation between the 2 and 1 norms,

$$
\mathrm{J}_{S} \geq \frac{\mathrm{J}_{A}^{2}}{(\mathrm{~N}-\nu)}
$$

we also have

$$
\begin{aligned}
\operatorname{SIR}(\mathrm{dB}) \leq & 10 \log _{10}\left(1+2 \sum_{\substack{0<\mathrm{m} \leq \nu\\
}}\left|\lambda_{\mathrm{MAX}, \mathrm{m}, \mathrm{N}}\right|^{2}\right)+ \\
& 10 \log _{10}(\mathrm{~N}-\nu)-2 \mathrm{~J}_{A}(\mathrm{~dB})
\end{aligned}
$$

Finally, since we have trivially that $\mathrm{J}_{A} \geq \mathrm{J}_{L}$, we have the bound

$$
\begin{aligned}
\operatorname{SIR}(\mathrm{dB}) \leq & 10 \log _{10}\left(1+2 \sum_{\substack{0<\mathrm{m} \leq \nu \\
0 \log _{10}(\mathrm{~N}-\nu)-2 \mathrm{~J}_{L}(\mathrm{~dB})}}\left|\lambda_{\mathrm{MAX}, \mathrm{m}, \mathrm{N}}\right|^{2}\right)+ \\
& 10 \log )
\end{aligned}
$$


Which shows that a high SLAM cost implies poor SIR performance. Unfortunately, the reverse implication does not hold, as we show in the next section.

\section{A. Implications for the Efficacy of the SLAM Design}

Here we show that the SLAM channel shortening metric suffers from inherent ambiguities whenever $\nu<\mathrm{N}-1$ which allow a globally minimum SLAM cost of 0 to be associated with combined responses which give arbitrarily bad performance SIR. This means that, despite the fact that SLAM has global minima at perfectly shortened locations, it will suffer from global minima which have arbitrarily bad performance down to SIR $=3 \mathrm{~dB}$. A particularly simple example of this phenomenon occurs for $\mathrm{c}(z)$ of the form

$$
\mathrm{c}(z)=\sqrt{a}+\sqrt{1-a} z^{-\mathrm{N}}
$$

which has SLAM cost of $-\infty \mathrm{dB}$ for any $a \in\left[\frac{1}{2}, 1\right)$ and $\nu<\mathrm{N}$, but has SIR at the matched filter output of $10 \log _{10}\left(\frac{1}{2 a(1-a)}\right) \mathrm{dB}$ which may be chosen arbitrarily large via choice of $a \in\left[\frac{1}{2}, 1\right)$.

\section{Performance And Convergence of Blind CHANNEL SHORTENERS}

The type of performance bounds in section IV are important for adaptive versions of the blind channel shortening designs, because they show whether or not (stochastic) gradient descents on the cost functions may be predicted, given a proper choice of a step size, to be accompanied with an increase in SIR performance at the matched filter output. In particular, the lack of a lower bound on the SIR performance at the output of the matched filter implies that adaptive channel shortening filters which minimize the SLAM cost with stochastic gradient descents, while they may be proved to converge to a minimum of the $J_{L}$ cost, can not be guaranteed to converge to channel shorteners which shorten the channel and give a good matched filter output SIR. By providing both upper and lower bounds on the SIR performance of a combined response $\mathrm{c}(z)$ in terms of its SSAM and SAAM, however, we have shown that a sufficiently large decrease in either of $J_{S}(\mathrm{~dB})$ or $J_{A}(\mathrm{~dB})$ is guaranteed to be accompanied with an increase in SIR (dB) performance at the output of a matched filter. More importantly, this increase in SIR (dB) scales in an affine manner with the decrease in $J_{S}(\mathrm{~dB})$ or $J_{A}(\mathrm{~dB})$. Indeed, suppose that the $\mathrm{J}_{S}$ cost of the initialization of the adaptive filter performing a stochastic gradient descent on $\mathrm{J}_{S}$ is $\mathrm{J}_{S}^{(0)}$ (dB), and that the $J_{S}$ cost after $T$ iterations has decreased to $\mathrm{J}_{S}^{(T)}(\mathrm{dB})$. One may then combine the bounds (2) and (3) to relate the corresponding change in the SIR performance $\Delta$ SIR (dB) with the formula

$$
\begin{aligned}
\Delta \mathrm{SIR}(\mathrm{dB}) \geq & \mathrm{J}_{S}^{(0)}(\mathrm{dB})-\mathrm{J}_{S}^{(T)}(\mathrm{dB})- \\
& 10 \log _{10}\left(1+2 \sum_{0<\mathrm{m} \leq \nu}\left|\lambda_{\mathrm{MAX}, \mathrm{m}, \mathrm{N}}\right|^{2}\right)
\end{aligned}
$$

A parallel argument for $\mathrm{J}_{A}$ yields

$$
\begin{aligned}
\Delta \mathrm{SIR}(\mathrm{dB}) \geq & 2 \mathrm{~J}_{A}^{(0)}(\mathrm{dB})-2 \mathrm{~J}_{A}^{(T)}(\mathrm{dB})-10 \log _{10}(\mathrm{~N}-\nu) \\
& -10 \log _{10}\left(1+2 \sum_{0<\mathrm{m} \leq \nu}\left|\lambda_{\mathrm{MAX}, \mathrm{m}, \mathrm{N}}\right|^{2}\right)
\end{aligned}
$$

This means that one can prove the convergence of the SIR performance of adaptive channel shorteners from the $J_{S}$ and $\mathrm{J}_{A}$ costs by proving the convergence of the corresponding stochastic gradient descents, e.g. by applying theory from [9].

\section{CONCLUSIONS}

In this paper we showed that matched filters are important in systems employing autocorrelation based channel shortening due to inherent ambiguities in the relationship between a signal and its autocorrelation. The use of the matched filter allowed us to provide clean, simple bounds on the signal to interference ratio at the output of the channel shortener in terms of the SSAM [4], SAAM [5], and SLAM [6] costs. We showed that a sufficiently large decrease in SSAM or SAAM cost always implies an increase in SIR performance, while the same can not be said for the SLAM design. This implies that the convergence of adaptive implementations of the SSAM and SAAM channel shorteners to good SIR performance may be studied by proving convergence (i.e. proper operation) of the stochastic gradient descents on SSAM and SAAM costs to minima of sufficient depth. Analysis and some analytical examples showed that studying the convergence of the SIR performance of an adaptive channel shortener on the the SLAM cost is more pathological, because even guaranteeing convergence of the SLAM cost to low values does not guarantee convergence to high SIRs.

\section{REFERENCES}

[1] D. D. Falconer and F. R. Magee, "Adaptive Channel Memory Truncation for Maximum Likelihood Sequence Estimation,” Bell Sys. Tech. Journal, pp. 1541-1562, Nov. 1973.

[2] P. J. W. Melsa, R. C. Younce, and C. E. Rohrs, "Impulse Response Shortening for Discrete Multitone Transceivers," IEEE Trans. on Comm., vol. 44, pp. 1662-1672, Dec. 1996.

[3] R. K. Martin, "Blind, Adaptive Equalization for Multicarrier Receivers," Ph.D. dissertation, Cornell University, Ithaca, NY, May 2004.

[4] J. Balakrishnan, R. K. Martin, and C. R. Johnson, Jr., "Blind, adaptive channel shortening by sum-squared auto-correlation minimization (SAM)," IEEE Trans. Signal Processing, vol. 51, pp. 3086-3093, Dec. 2003.

[5] R. Nawaz and J. A. Chambers, "Robust blind channel shortening in impulsive noise environments," in EUSIPCO, Vienna, Austria, 2004.

[6] R. Nawaz and J. Chambers, "Blind adaptive channel shortening by single lag auto-correlation minimization (SLAM)," Electronics Letters, vol. 40, pp. 1609 - 1610, Dec. 2004.

[7] S. Barbarossa, G. Scutari, and A. Swami, "MUI-free CDMA Systems Incorporating Space-Time Coding and Channel Shortening," in Proc. International Conference on Acoustics, Speech, and Signal Processing (ICASSP), vol. 3, May 2002, pp. 2213-2216.

[8] J. M. Walsh, R. K. Martin, A. G. Klein, N. S. Xenias, J. A. Pagnotta, and C. R. Johnson, Jr., "Necessary and sufficient conditions for perfect channel shortening and implications for interference mitigation," in Proc. $V$ IEEE Signal Processing Workshop on Signal Processing Advances in Wireless Communications (SPAWC), Lisbon, Portugal, July 2004.

[9] V. Solo and X. Kong, Adaptive Signal Processing Algorithms: Stability and Performance. Englewood Cliffs, N.J.: Prentice Hall, 1995. 